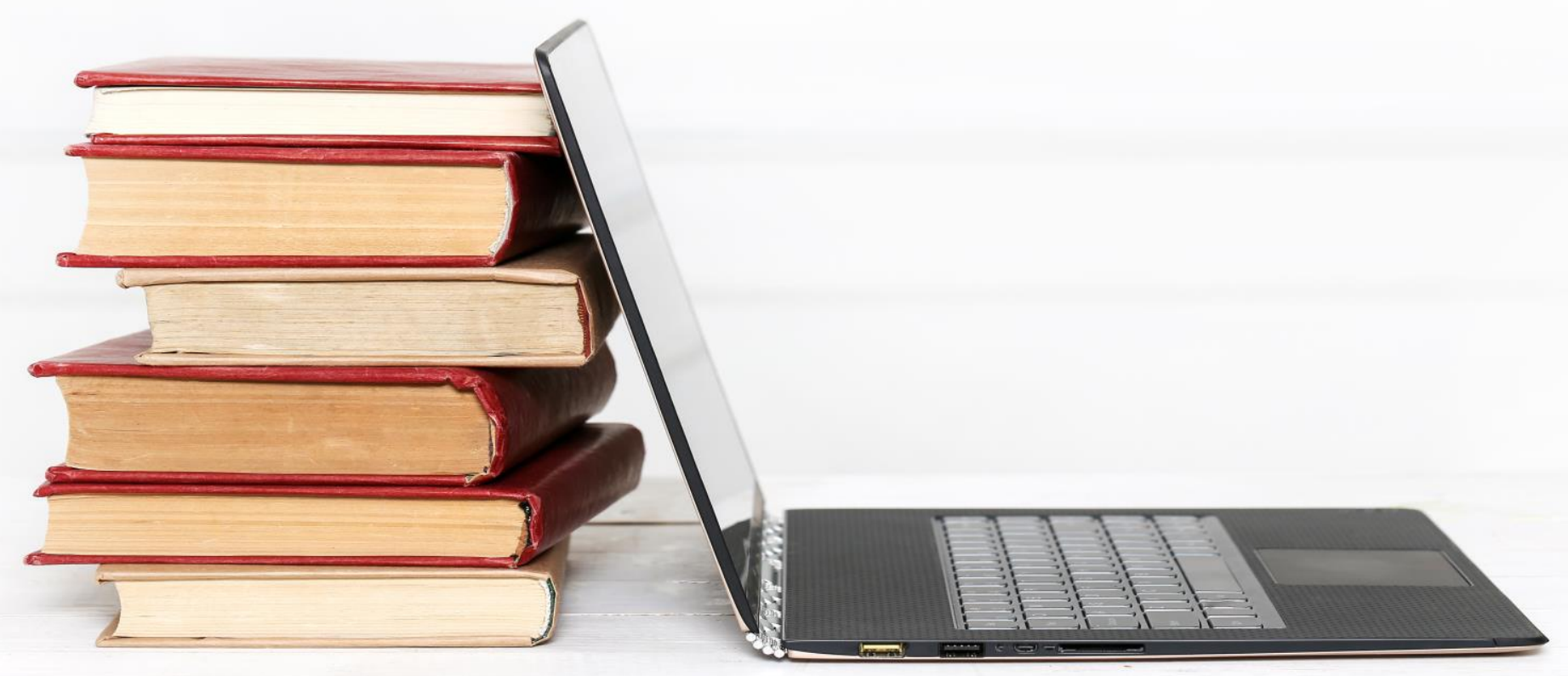

(c) Diseñado por Racool_studio / Freepik. Fuente: https://www.freepik.es/foto-gratis/libros-laptop_7220901.htm

\title{
El ambiente virtual en la Universidad Veracruzana
}

\section{Angélica María Hernández Ramírez ${ }^{\mathrm{a}}$}

RESUMEN: El acceso a la educación superior parece ser un privilegio debido a la gran demanda y limitada infraestructura. Se evaluó el ambiente virtual como innovación educativa y la innovación educativa en el ambiente virtual en la Universidad Veracruzana. Se revisaron los programas educativos dentro del Área de Formación de Elección Libre y se estimaron los indicadores de género, acceso y cobertura de un programa educativo virtual. El ambiente virtual representó el 8\% de la innovación educativa. Cinco regiones tuvieron acceso al programa (estudio de caso), alcanzando el 57\% de cobertura en la universidad. La innovación en la práctica pedagógica asociada al uso de las Tecnologías de la Información y Comunicación es clave para una innovación educativa en los ambientes virtuales. La educación virtual se reconoce como estrategia incluyente que permitiría afrontar la demanda educativa a nivel nacional.

\footnotetext{
a Investigadora de tiempo completo, académica de carrera Titular "C", Centro de EcoAlfabetización y Diálogo de Saberes, Universidad Veracruzana. Contacto: angehernandez@uv.mx. ORCID: http://orcid.org/0000-00032042-8496.
} 
Artículo - Humanidades

Palabras clave: Ambiente virtual; criterios de evaluación; educación superior; diseño de programas educativos.

\begin{abstract}
Access to higher education seems to be a privilege due to high demand and limited infrastructure. Virtual environment as educational innovation and educational innovation in the virtual environment were assessed at the University of Veracruz. The educational programs into Training of Free Choice Area were reviewed, whereas the gender, access and coverage indicators of a virtual educational program were estimated. The virtual environment represented $8 \%$ of educational innovation. Five regions had access to the program (study case), reaching 57\% of coverage at the university. Innovation in pedagogical practice linked to the use of Information and Communication Technologies is key for an educational innovation in the virtual environment. This research recognizes to the virtual education as inclusive strategy that would allow to face the educational demand at national level.
\end{abstract}

Keywords: Design of Educational Program; Evaluation Criteria; Higher Education; Virtual Environment

\title{
Introducción
}

工

a creciente demanda educativa de los jóvenes aspirantes a cursar una carrera profesional asociada a una realidad de no contar con los espacios educativos suficientes (infraestructura) propicia que la educación superior en México se siga considerando como un bien privilegiado a nivel nacional. No obstante, al ser la educación un derecho constitucional para todos, la educación deberá asentarse en los principios de inclusión, permanencia y continuidad. (Cámara de Diputados, 2019)

La Universidad Veracruzana (UV) es una Institución de Educación Superior Pública en México con cinco regiones y cuatro sedes distribuidas a lo largo del estado de Veracruz. La institución se caracteriza por presentar un Modelo Educativo Integral y flexible (MEIF), el cual es centrado en el aprendizaje y formación del estudiante con base en el principio de pertinencia social, transversalidad, integridad y flexibilidad (Hernández-Ramírez, 2016, 2018; Universidad Veracruzana, 2017). Dentro del diseño curricular institucional, el Área de Formación de Elección Libre (AFEL) ofrece un conjunto de cursos que incorporan diversas actividades provenientes de las artes, el deporte y las derivadas de las distintas disciplinas con miras a 
fortalecer la formación integral de los estudiantes. Por sus características, la educación se entiende como un proceso de enseñanza-aprendizaje y los cursos se reconocen como Experiencias Educativas (EE) a nivel institucional. (Hernández-Ramírez, 2016, 2018)

El MEIF se ha ido implementado y diversificando de manera paulatina en función de las demandas y posibilidades de la planta docente y de la infraestructura universitaria. Destaca la incorporación de investigadores como docentes a nivel licenciatura (no sólo a nivel de posgrado) y el desarrollo de "Eminus Sistema de Educación Distribuida", como plataforma institucional de distribución y apoyo al proceso de enseñanza-aprendizaje a distancia (ambiente virtual).

La oferta académica virtual se basa en el uso de las tecnologías de la información y de la comunicación (TICs). En particular, el uso de TICs en los programas educativos por sí mismos representan una propuesta innovadora a nivel de educación superior en México (Munévar García, Lasso Cárdenas \& Rivera Piragua, 2015; Niño Carrasco, Castellanos Ramírez \& Viloria Hernández, 2019). No obstante, y desde el punto de vista de innovación educativa en educación superior; el diseño de la propuesta educativa en modalidad virtual debe partir del entendimiento de quiénes son los usuarios de las TICs (situar al usuario ante la herramienta) y los propósitos del uso de las TICs (proceso de enseñanza-aprendizaje) en el ambiente universitario. (Hernández-Ramírez, 2016; Niño Carrasco, Castellanos Ramírez \& Viloria Hernández, 2019; George Reyes \& Salgado Rodríguez, 2019)

En este sentido, el diseño de la propuesta educativa debe asentarse claramente en un modelo pedagógico que potencialice las posibilidades de la herramienta misma y de la experiencia derivada de la interacción individual y grupal en el ambiente virtual (Berridi Ramírez \& Martínez Guerrero, 2017). En particular, el programa de la EE virtual "Interacciones plantaanimal: ecología y conservación" se diseñó desde la propuesta pedagógica constructivista con actividades dirigidas al desarrollo y evaluación de las competencias de los estudiantes matriculados (Hernández-Ramírez, 2016).

Específicamente, se trabajó con base en el desarrollo del eje conceptual (teóricoepistemológico) a partir de la lectura, análisis e identificación de conceptos clave de lecturas seleccionadas (lectoescritura, Suárez Cárdenas, Pérez Rodríguez, Vergara Castaño \& Alférez Jiménez, 2015). Por otro lado, el eje procedimental (heurístico) se trabajó a través de la elaboración de ensayos basados en investigaciones realizadas por los propios estudiantes acorde a los temas revisados en el curso (Hernández-Ramírez, 2016, 2018). Finalmente, el eje actitudinal (axiológico) se trabajó a través de actividades dirigidas en foros de discusión. 
Desde esta perspectiva, el estudiante reconoce y se apropia de un discurso, lo que le permite establecer un diálogo de manera horizontal (pares académicos), y promover un proceso de autorregulación y aprendizaje colectivo o social (Hernández-Ramírez, 2016, 2018; Berridi Ramírez \& Martínez Guerrero, 2017). A partir de esta propuesta pedagógica se reconoce el papel central que tiene el estudiante en su proceso de enseñanza-aprendizaje. (autogestión del conocimiento; Berridi Ramírez \& Martínez Guerrero, 2017)

La presente investigación parte del análisis de dos vertientes de la innovación educativa del ambiente virtual en la Universidad Veracruzana. Específicamente se investigó: 1) el ambiente virtual como innovación educativa en la universidad y 2) la innovación educativa en el ambiente virtual a través del análisis del estudio de caso de la EE "Interacciones plantaanimal: ecología y conservación".

\section{Metodología}

Se realizó una búsqueda, sistematización, revisión y análisis de la oferta educativa perteneciente al AFEL dentro del MEIF de la Universidad Veracruzana para investigar y analizar al ambiente virtual como innovación educativa en la institución y se identificaron las siguientes variables a analizar: 1) Modalidad de la EE (presencial o virtual), 2) Número de créditos, 3) Año de creación del Programa, 4) información no disponible asociada con la custodia de datos (i.e., registro o carencia de la información en los programas de las EE y/o quiénes otorgan/niegan acceso a la información de programa completo). La segunda vertiente se investigó a través de la pertinencia de la innovación educativa propuesta en la EE virtual "Interacciones planta-animal: ecología y conservación" en concordancia con el MEIF. Para ello, se reportan los resultados de los indicadores de género, acceso y cobertura de la EE virtual antes mencionada en el periodo semestral febrero-julio 2019. (Hernández-Ramírez, 2016)

\section{Resultados}

La Universidad Veracruzana oferta 374 EE dentro del AFEL para atender la matrícula de estudiantes distribuidos en cinco regiones y cuatro sedes a lo largo del estado de Veracruz. Del total de EE ofertadas en la institución, 355 son en modalidad presencial y 19 son en 
modalidad de ambiente virtual. En la Tabla 1 se observa que la oferta educativa se distribuye en siete categorías académicas con base en la orientación reconocida en la institución y con un número variado de créditos y horas asociadas a las distintas EE. Debido a que no se tuvo acceso al $45 \%$ de los programas de las EE ofertadas en la institución, se procedió a trabajar con la información contenida en 204 EE.

Tabla 1

Oferta Educativa asignada al Área de Formación de Elección Libre (AFEL) del Modelo Educativo Integral y Flexible (MEIF) de la Universidad Veracruzana

\begin{tabular}{|l|l|l|l|l|}
\hline $\begin{array}{l}\text { Clasificación } \\
\text { Académica }\end{array}$ & $\begin{array}{l}\text { Experiencias } \\
\text { Educativas } \\
\text { (EE) }\end{array}$ & $\begin{array}{l}\text { Ambiente } \\
\text { virtual } \\
\text { (proporción) }\end{array}$ & $\begin{array}{l}\text { Número de } \\
\text { créditos } \\
\text { (intervalo) }\end{array}$ & $\begin{array}{l}\text { Custodia de datos } \\
\text { (Disponibilidad/acceso } \\
\text { en porcentaje) }\end{array}$ \\
\hline Salud Integral & 66 & 0 & $3-6$ & 94 \\
\hline Idiomas & 129 & 0 & $5-8$ & 3 \\
\hline $\begin{array}{l}\text { Formación y } \\
\text { divulgación } \\
\text { científica }\end{array}$ & 41 & 0 & $4-10$ & 85 \\
\hline $\begin{array}{l}\text { Manifestaciones } \\
\text { artísticas }\end{array}$ & 44 & 2 & $3-10$ & 73 \\
\hline $\begin{array}{l}\text { Innovación } \\
\text { Educativa }\end{array}$ & 24 & 29 & $2-6$ & 92 \\
\hline Ecología & 21 & 5 & $4-7$ & 81 \\
\hline $\begin{array}{l}\text { Cultura } \\
\text { ciudadana }\end{array}$ & 49 & 20 & $4-10$ & 67 \\
\hline
\end{tabular}

Fuente: Creación propia. 


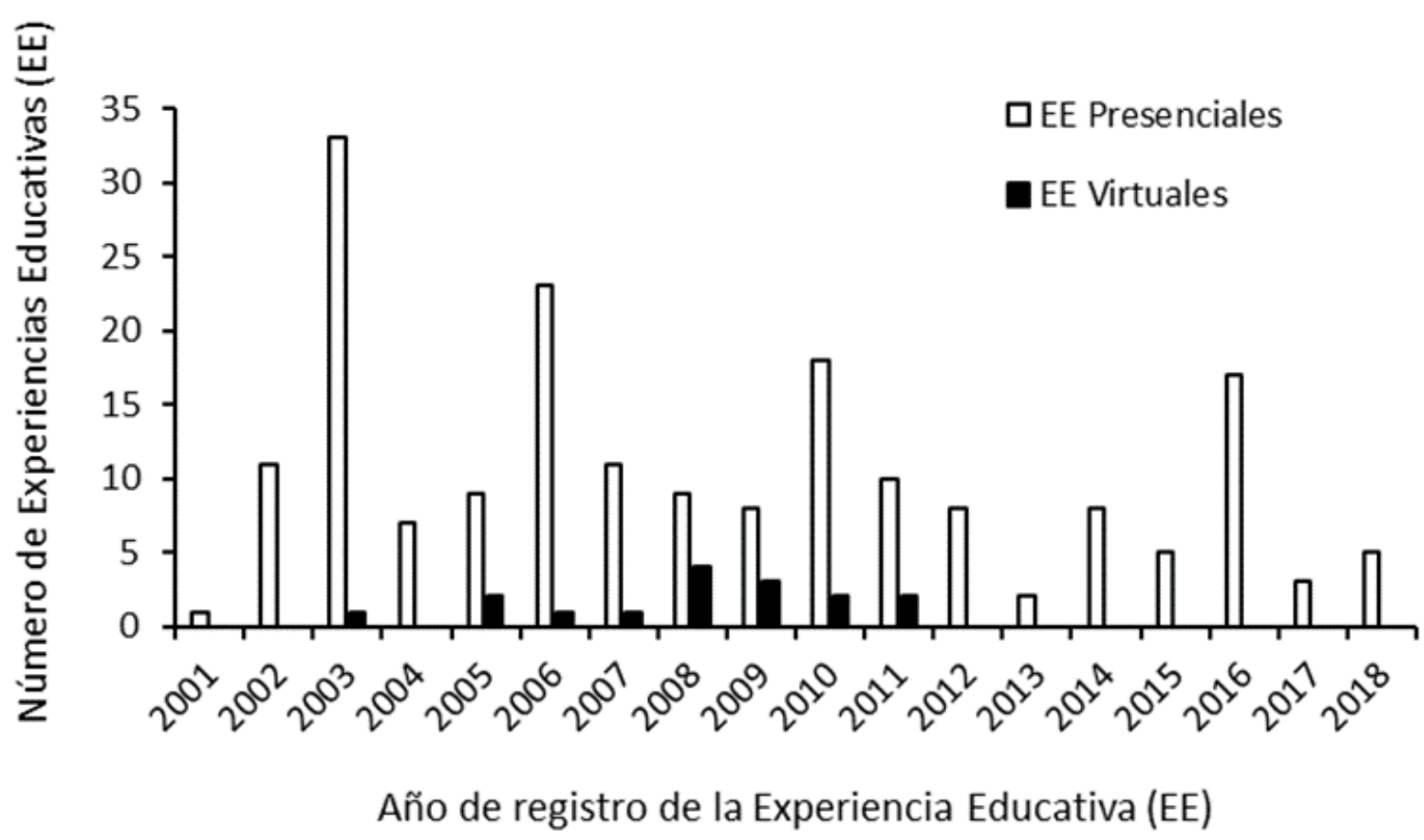

Figura 1. Oferta educativa en el Área de Formación de Elección Libre (AFEL) en la Universidad Veracruzana.

En la Figura 1 se observa que el registro de EE dentro del AFEL inició con una EE registrada en el 2001 y en 2018 se registraron las últimas cinco EE. En 2001 se registró la primera EE en modalidad de ambiente virtual y en 2011 se registraron las últimas dos EE en esta modalidad. Las EE en modalidad de ambiente virtual representa el 8\% de innovación a la oferta académica de la institución.

En lo que respecta a la innovación educativa en el ambiente virtual, los resultados muestran que en total se inscribieron 34 alumnos (18 mujeres y 16 hombres; 1:1 proporción sexual) a la EE virtual "Interacciones planta-animal: ecología y conservación" en el periodo de estudio. De éstos, tres alumnos no se presentaron en el ambiente virtual (9\%). En términos generales, se presentó una permanencia del 74\% de los alumnos, por lo que hubo una deserción del $26 \%$ de los alumnos inscritos. La pertinencia (repercusión de la innovación educativa) de la EE virtual "Interacciones planta-animal: ecología y conservación" medida a través del indicador de acceso mostró que la EE se oferta y llega a las cinco regiones de la Universidad con base en la 
Tabla 2. Específicamente, la EE tiene una mayor demanda en la región de Veracruz-Boca del Río y una menor demanda en Poza Rica-Tuxpan.

Tabla 2

Indicadores de pertinencia de la EE virtual "Interacciones planta-animal: ecología y conservación" organizados a nivel de acceso en la Universidad Veracruzana

\begin{tabular}{|l|l|l|l|l|l|}
\hline \multicolumn{1}{|c|}{ Región } & $\begin{array}{l}\text { Total de } \\
\text { alumnos }\end{array}$ & $\begin{array}{l}\text { Número } \\
\text { mujeres }\end{array}$ & $\begin{array}{l}\text { Número } \\
\text { hombres }\end{array}$ & $\begin{array}{l}\text { Permanencia } \\
(\%)\end{array}$ & $\begin{array}{l}\text { Deserción } \\
(\%)\end{array}$ \\
\hline $\begin{array}{l}\text { Coatzacoalcos- } \\
\text { Minatitlán }\end{array}$ & 5 & 3 & 2 & 80 & 20 \\
\hline Orizaba-Córdoba & 5 & 2 & 3 & 60 & 40 \\
\hline Poza Rica-Tuxpan & 4 & 4 & 0 & 50 & 50 \\
\hline Veracruz-Boca del Río & 10 & 6 & 4 & 100 & 0 \\
\hline Xalapa & 7 & 1 & 6 & 57 & 43 \\
\hline
\end{tabular}

En la Tabla 3 se observó que la EE tiene una cobertura del 57\% a nivel institucional, ya que cubre cuatro de las siete áreas académicas reconocidas por la institución. En específico, estudiantes pertenecientes a 12 carreras diferentes se inscribieron a la EE virtual "Interacciones planta-animal: ecología y conservación" durante el periodo de estudio.

Tabla 3.

Indicadores de pertinencia de la EE virtual "Interacciones planta-animal: ecología y conservación" organizados a nivel de cobertura en la Universidad Veracruzana

\begin{tabular}{|l|l|l|l|l|l|}
\hline Área académica & $\begin{array}{l}\text { Total de } \\
\text { alumnos }\end{array}$ & $\begin{array}{l}\text { No. } \\
\text { mujeres }\end{array}$ & $\begin{array}{l}\text { No. } \\
\text { hombres }\end{array}$ & $\begin{array}{l}\text { Permanencia } \\
(\%)\end{array}$ & $\begin{array}{l}\text { Deserción } \\
(\%)\end{array}$ \\
\hline $\begin{array}{l}\text { Biológico- } \\
\text { Agropecuaria }\end{array}$ & 5 & 3 & 2 & 100 & 0 \\
\hline Ciencias de la Salud & 17 & 3 & 14 & 76 & 24 \\
\hline Humanidades & 3 & 2 & 1 & 67 & 33 \\
\hline Técnica & 6 & 2 & 4 & 50 & 50 \\
\hline
\end{tabular}

\section{Discusión}

El MEIF se estableció de manera paulatina en la Universidad Veracruzana a partir de 1998 en coherencia con el Plan Nacional de Desarrollo y el Programa Nacional de Educación 2001-2006 del país (Secretaría de Educación Pública, 2001). El MEIF al estar centrado en la formación 


\section{Artículo - Humanidades}

integral, transversal y flexible del estudiante ha propiciado una demanda en la oferta educativa en la institución (Universidad Veracruzana, 2017). En respuesta a esto, la Universidad Veracruzana innovó su propio modelo educativo al incorporar el AFEL. En este sentido, el AFEL aporta a los estudiantes 374 EE como opciones electivas para cubrir sus créditos formativos.

La Universidad Juárez Autónoma de Tabasco cuenta con un Modelo Educativo Flexible similar al de la Universidad Veracruzana y ofrece un área electiva de 32 cursos, talleres y seminarios para que los estudiantes puedan cubrir sus créditos (Universidad Juárez Autónoma de Tabasco, 2019). La Universidad Anáhuac cuenta con un Modelo Educativo de Formación Integral Semiflexible, por lo que ofrece 32 cursos dentro del área de formación integral, 26 cursos dentro del área de humanidades y 54 cursos dentro del área de escuelas y facultades (Universidad Anáhuac, 2020). Lo anterior muestra la importancia de incorporar el Área Electiva como estrategia de flexibilidad dentro de los programas de estudio de las Universidades en México.

A través de un proceso de evaluación del MEIF (2015-2017), la institución reportó una respuesta favorable (82\%) por parte de los estudiantes para optar por los cursos AFEL, mientras que el 70\% de los estudiantes encuestados reportó haber cursado una EE con contenidos distintos a los de su formación disciplinar (Alarcón Montiel, Rosales Rodríguez \& Ortega Guerrero, 2011). Por lo anterior, el AFEL parece trabajar en cumplimiento de la formación integral y transversal del alumno acorde con los principios del MEIF.

Desde la perspectiva de los alumnos, la flexibilidad curricular asociada a la oferta académica les genera horarios discontinuos y gastos adicionales relacionados con la necesidad que tienen de trasladarse de un plantel educativo a otro (Colorado Lara, 2018). Aunado a esto, la capacidad de atender a un número limitado de estudiantes acorde con la infraestructura disponible ha propiciado un rezago del 17\% en el tiempo de titulación de los estudiantes cuando se comparó con estudiantes que cursaron el anterior modelo educativo universitario. (Colorado Lara, 2018; Universidad Veracruzana, 2017)

En este sentido, la oferta académica en el ambiente virtual innovó al modelo educativo al establecer nuevas formas de aprender y de enseñar a través del uso de las tecnologías de la información y de la comunicación (TICs) (Hernández-Ramírez, 2016; Niño Carrasco, Castellanos Ramírez \& Viloria Hernández, 2019). El análisis de la evaluación de MEIF a nivel institucional reconoció que las EE en modalidad virtual contribuyeron a la resolución de los 
problemas asociados a los gastos de movilidad y limitada infraestructura (Universidad Veracruzana, 2017). No obstante, existe un sector de la población universitaria que no tienen acceso a las tecnologías de la información y de la comunicación, por lo que ese sector queda excluido de esa oferta educativa (Hernández-Ramírez, 2016). Dicho sector corresponde a la Universidad Veracruzana Intercultural con sus sedes en la Huasteca, Totonacapan, Grandes Montañas y Las Selvas (26\% de la matrícula escolar).

A nivel educativo, los procesos pedagógicos en el ambiente virtual se apartan de los modelos educativos tradicionales, ya que parten de un proceso de enseñanza-aprendizaje basado en la autonomía y regulación (individual y colectiva) del propio estudiante, por lo que el estudiante adquiere consciencia, responsabilidad y apropiación de su propio proceso (i.e., construcción del conocimiento; Hernández-Ramírez, 2016, 2018; Niño Carrasco, Castellanos Ramírez \& Viloria Hernández, 2019; George Reyes \& Salgado Rodríguez, 2019).

Aunado a lo anterior, la educación en el ambiente virtual tiende a caracterizarse por grupos de estudiantes heterogéneos tanto en su proceso formativo (disciplina o carrera que están cursando), etapa de su proceso formativo (semestre), contextos (regiones) e intereses particulares (Hernández-Ramírez, 2016; Universidad Veracruzana, 2017). La heterogeneidad en los grupos demanda un diseño curricular que sea incluyente y justo en los criterios de evaluación, independientemente de la disciplina de la cual provengan los estudiantes. En este sentido, la EE virtual "Interacciones planta-animal: ecología y conservación" innovó y aportó al MEIF el enfoque de trabajo pedagógico de tipo constructivista apoyado en el desarrollo y evaluación de las competencias individuales y colectivas de los estudiantes matriculados. (Hernández-Ramírez, 2016; Berridi Ramírez \& Martínez Guerrero, 2017)

A partir de esta propuesta educativa se desprenden nuevas formas de ver, entender, construir y evaluar el conocimiento (i.e., desempeño del estudiante). Es decir, la EE aporta elementos que ejemplifican lo forma en la cual se vincula el proceso pedagógico con el uso de las TICs en los ambientes educativos de educación superior (Munévar García, Lasso Cárdenas \& Rivera Piragua, 2015; Niño Carrasco, Castellanos Ramírez \& Viloria Hernández, 2019; George Reyes \& Salgado Rodríguez, 2019). Específicamente, el desarrollo de actitudes, aptitudes y habilidades dirigidas a la construcción de conocimientos y re-significación de los mismos hace del programa educativo una experiencia abierta, flexible e incluyente. (Hernández-Ramírez, 2016)

Lo anterior se corrobora al visibilizar el alcance que la EE virtual "Interacciones plantaanimal: ecología y conservación" tiene a nivel institucional en términos de acceso y cobertura. Al ser el desarrollo de las competencias una tarea viable de alcanzar desde el punto de vista 
del estudiante, el proceso educativo/evaluativo se vuelve alcanzable, justo, pertinente y equilibrado para el estudiante. En este sentido, la propuesta educativa en el ambiente virtual acentúa y promueve el desarrollo de las habilidades procedimentales. Del mismo modo, el modelo educativo promueve los valores éticos ligados a la construcción pertinente y autónoma del conocimiento (eje teórico). Dado que la construcción del conocimiento se adapta a las necesidades disciplinares y contextuales particulares de cada estudiante, este modelo de enseñanza y aprendizaje es afín al modelo educativo centrado en el estudiante de la institución (MEIF).

\section{El ambiente educativo virtual como medio de inclusión social}

La noción de inclusión social emerge como parte de las políticas púbicas dirigidas a contrarrestar los problemas de discriminación y desigualdad en la nación (Cámara de Diputados, 2018). La discriminación posee muchas dimensiones e impacta el bienestar de los mexicanos en diferentes ámbitos de su vida, por lo que su falta de atención genera inequidad y rezago en el país. En este sentido, los principios que acompañan a la política pública de igualdad de oportunidades e inclusión social incluyen: equidad, igualdad de oportunidades, autonomía individual, libertad de decisión, derecho a participar, respeto a la diferencia, accesibilidad, igualdad entre hombres y mujeres, transversalidad y no discriminación. (Artículo 5, Cámara de Diputados, 2018)

Desde el punto de vista educativo, el ambiente virtual ha sido un tema en debate, ya que el derecho a la educación a la cual aspiran todos los mexicanos se enfrenta a una realidad en la cual existen claras diferencias de acceso a un ambiente educativo virtual (i.e., conjunto tecnológico que incluye dispositivos y redes de telecomunicación). Específicamente, la "brecha digital" considera aspectos relacionados con el acceso, uso y apropiación de las TICs por parte de la población como medio de comunicación (INEGI 2020). Con base en los indicadores sobre disponibilidad y uso de TICs a nivel nacional, se sabe que cerca del 50\% de la población posee computadora y acceso a internet en sus hogares (44.3 - 45.6\%; población con computadora y 44.3-45.6 \%, población con conexión a internet; periodo evaluado de 2016 al 2019; INEGI, 2020). De ese porcentaje, la mitad de la población (una cuarta parte) utiliza estos medios como herramienta de apoyo escolar (44.6 -52.2\%; periodo evaluado de 2016 al 2019 ; INEGI, 2020). Paralelamente, se reporta que del 73.3 al 75.1\% de la población son usuarios de teléfonos celulares (INEGI, 2020). Por ello, es importante reconocer que en la actualidad 
existen otros dispositivos (nuevas tecnologías) que pueden incorporarse a el ambiente escolar, por lo que contribuiría a disminuir la brecha digital y promover la inclusión social.

El Instituto Federal de Telecomunicaciones (2018) reportó que la población que es residente y vive en las ciudades presentan una mayor probabilidad de utilizar internet (25\%) con respecto a aquellos habitantes que viven fuera de la ciudad. Si bien existen obvias diferencias en lo que se refiere a acceso, uso y apropiación de las TICs entre zonas rurales y urbanas en el país, esta diferencia se suma a una problemática de marginación que se viven a nivel nacional en el sector educativo (infraestructura, docentes y una mayor oferta educativa disponible en las ciudades). Por lo que las TICs se constituyen como un componente adicional de desigualdad y no como un factor de exclusión social per se.

En el contexto meramente educativo, el uso de las TICs como herramienta de trabajo constituye una estrategia para que los jóvenes tengan las mismas oportunidades de elegir y cursar distintos programas educativos a distancia y de forma no presencial. Aunado a esto, los recursos educativos que se emplean en el ambiente virtual incluyen textos, videos, bibliotecas virtuales, literatura especializada y de calidad de libre acceso, dentro y fuera de la institución (Suárez Cárdenas, Pérez Rodríguez, Vergara Castaño \& Alférez Jiménez, 2015; Hernández-Ramírez, 2016). Por lo anterior, este tipo de trabajo y de diseño curricular representa una propuesta emergente de igualdad de condiciones y recursos educativos para los estudiantes que optan por este tipo de educación. A su vez, la educación a distancia está diseñada para que los estudiantes (usuarios) tengan una flexibilidad en los horarios y lugares de trabajo (Hernández-Ramírez, 2016; Colorado Lara, 2018; Niño Carrasco, Castellanos Ramírez \& Viloria Hernández, 2019). En este sentido, la educación a distancia acerca la escuela a los usuarios y a sus realidades/necesidades particulares (i.e., condiciones familiares, económicosociales y de salud). En conjunto, el ambiente educativo virtual actúa como medio de inclusión social, ya que contribuye a atender los principio que acompañan la política publica de igualdad de oportunidades e inclusión social previamente citados.

\section{Conclusiones}

La oferta académica en el ambiente virtual innovó al modelo educativo al establecer nuevas formas de aprender y de enseñar a través del uso de las tecnologías de la información y de la comunicación (TICs). La EE virtual "Interacciones planta-animal: ecología y conservación" ejemplifica un caso de innovación en los procesos y modelos pedagógicos empleados en ambientes virtuales (educación a distancia) y en grupos heterogéneos. A través del estudio de 


\section{Artículo - Humanidades}

caso se ejemplifica la forma en cómo la innovación en la práctica pedagógica se une a la innovación en el uso de las TICs en la educación superior. Esta investigación realza la importancia que tiene el reconocer a la oferta educativa virtual adscrita a las Instituciones de Educación Superior (IES) como estrategia incluyente, pertinente y justa que permita afrontar la creciente demanda educativa de los jóvenes a nivel nacional, lo cual es un derecho establecido a nivel constitucional.

\section{Referencias}

Alarcón Montiel, E., Rosales Rodríguez, O. A. y Ortega Guerrero, J. C. (2011). El impacto del Modelo Educativo Integral y Flexible de la Universidad Veracruzana en las trayectorias escolares. El caso del Área Académica Técnica Región Xalapa. XI Congreso Nacional de Investigación Educativa. https://bit.ly/2KjeOaM.

Berridi Ramírez, R. y Martínez Guerrero, J. I. (2017). Estrategias de autorregulación en contextos virtuales de aprendizaje. Perfiles Educativos, XXXIX (156): 89-102.

Cámara de Diputados. (2018). Ley General para la inclusión de las personas con discapacidad.

Diario Oficial de la Federación, 12 Julio 2018. https://www.dof.gob.mx.

Cámara de Diputados. (2019). Constitución Política de los Estados Unidos Mexicanos, Diario Oficial de la Federación, 9 de agosto de 2019. https://www.dof.gob.mx

Colorado Lara, S. (2018). El MEIF desde el punto de vista del estudiante. Tesis Licenciatura. Universidad Veracruzana.

George Reyes, C. E. y Salgado Rodríguez, L. I. (2019). Competencias investigativas con el uso de las TIC en estudiantes de doctorado. Apertura, 11(1): 40-55.

Hernández-Ramírez, A. M. (2016). Tendencias de género, acceso y cobertura de una experiencia educativa virtual transversal en la Universidad Veracruzana, en Moral JAH y Urquidi MLW (eds.), Tendencias y desafíos en la innovación educativa: un debate abierto (pp. 1227-1234). Universidad Veracruzana.

Hernández-Ramírez, A. M. (2018). El hilo en el laberinto: recorrido por la valoración y apropiación de los espacios educativos. En Paradowska, KB (ed.), ¡Ponte Trucha!, Talleres de arte y buen vivir en Rancho Viejo (pp. 12-22) Cuaderno EcoDialogo 7, Centro de EcoAlfabetización y Diálogo de Saberes, México.

INEGI. (2020). Encuesta Nacional sobre Disponibilidad y Uso de TIC en hogares, ENDUTIH. Instituto Nacional de Estadística y Geografía, México. https://www.inegi.org.mx Instituto Federal de Telecomunicaciones. (2018). Adopción de las TIC y sus de Internet en México. Impacto de las características sociodemográficas de los usuarios. México. http://www.ift.org.mx 
Munévar García, P. A., Lasso Cárdenas, E. P. y Rivera Piragua, J. A. (2015). Articulación entre modelos, enfoques y sistemas en educación en la virtualidad. Revista Virtual Universidad Católica del Norte, 46: 21-38.

Niño Carrasco, S. A., Castellanos Ramírez, J. C. y Viloria Hernández, E. (2019). Construcción del conocimiento y regulación del aprendizaje en tareas colectivas asíncronas. Apertura 11(1): 6-23.

Secretaría de Educación Pública. (2001). Programa Nacional de Educación 2001-2006. México. Diario Oficial de la Federación 15 enero 2003. https://www.dof.gob.mx

Suárez Cárdenas, A. I., Pérez Rodríguez, C. Y., Vergara Castaño, M. M. y Alférez Jiménez, V. H. (2015). Desarrollo de le lectoescritura mediante TIC y recursos educativos abiertos. APERTURA Revista de Innovación Educativa, 7(1): 1-13.

Universidad Anáhuac. (2020). Modelo Educativo Anáhuac. Cursos y Talleres. https://bit.ly/2yoH1Kq

Universidad Juárez Autónoma de Tabasco. (2019). Oferta Educativa. Cursos y Talleres. http://www.ujat.mx/287

Universidad Veracruzana. (2017). Evaluación del MEIF 2015-2017: Resultado de las Áreas de Formación Disciplinar, Termina, de Elección Libre y de la Tutoría Académica en el Modelo Educativo Integral y Flexible. Universidad Veracruzana. https://bit.ly/2VjOzJc 